Int. J. Dev. Biol. 53: 1069-1074 (2009)

doi: $10.1387 / \mathrm{ijdb} .082820 \mathrm{mg}$

\title{
Expression analysis and essential role of the putative tyrosine phosphatase His-domain-containing protein tyrosine phosphatase (HD-PTP)
}

\author{
MARIE-CLAUDE GINGRAS, DMITRI KHARITIDI, VALÉRIE CHÉNARD, NORIKO UETANI, \\ MAXIME BOUCHARD, MICHEL L. TREMBLAY and ARNIM PAUSE* \\ Goodman Cancer Centre and Department of Biochemistry, McGill University, Montréal, Québec, Canada
}

\begin{abstract}
The putative tyrosine phosphatase HD-PTP, encoded by the protein-tyrosine-phosphatase-n23 (Ptpn23) gene, has been described as a tumor suppressor candidate gene. However, its physiological roles and detailed expression profiles are poorly defined. To investigate HD-PTP functions, we generated a mouse model in which the Ptpn23 locus was disrupted by an in-frame insertion of a $\beta$-galactosidase-neomycin-phosphotransferase II ( $\beta$-geo) cassette. This insertion leads to the expression of a catalytically inactive truncated protein preserving only the uncharacterized N-terminal BRO1-like domain in fusion with $\beta$-geo under the control of the endogenous promoter. Here we report that homozygous gene deletion is lethal around embryonic day 9.5, suggesting that Ptpn23 is an essential requirement for early stages of embryonic development. Taking advantage of the $\beta$-galactosidase insertion into the Ptpn23 locus, we define the precise Ptpn23 expression pattern by performing $X$-gal staining at different stages of mouse development. Our results show that Ptpn23 is expressed early during mouse development and that its expression is maintained in adult tissues, markedly in the epithelial cells of many organs.
\end{abstract}

KEY WORDS: HD-PTP, Ptpn23, gene-trap, expression pattern, embryonic lethality

The protein-tyrosine-phosphatase-n23 (PTPN23) gene is a tumor suppressor gene candidate encoding a poorly characterized protein tyrosine phosphatase called His-domain-containing protein tyrosine phosphatase (HD-PTP) (Cao et al., 1998; Toyooka et al., 2000). The PTPN23 gene is located on the 3p21.3 tumor suppressor gene cluster frequently deleted in human kidney, breast, lung and cervical tumors and its overexpression inhibits ras-mediated transformation of NIH-3T3 cells (Cao et al., 1998; Toyooka et al., 2000, Imreh et al., 2003; Ji et al., 2005; Hesson et al., 2007). The HD-PTP protein encompasse four main domains: a functionally uncharacterized BRO1like domain (BRO), a histidine-rich domain (HIS), a classical protein tyrosine phosphatase domain (PTP) and a proteolytic degradation targeting sequence (PEST motif) (Toyooka et al., 2000). Potential functions for HD-PTP in inhibition of endothelial migration and EGFR endosomal trafficking were recently reported, but its role in vivo is still undefined (Castiglioni et al., 2007; Doyotte et al., 2008; Mariotti et al., 2008; Miura et al., 2008). The high level of conservation of HD-PTP among multi- cellular organisms (human, monkey, mouse, rat, chicken, frog and fly) suggests a significant and common biological function, as does its ubiquitous mRNA expression in adult human and rat organs (Cao et al., 1998; Mariotti et al., 2006). However, the developmental and cell type specific expression pattern remains unreported.

In order to verify the physiological functions of HD-PTP, we generated a mouse model containing a $\beta$-galactosidase-neomycin-phosphotransferase II ( $\beta$-geo) insertion into the Ptpn23 locus, leading to the expression of a catalytically inactive truncated protein in fusion with $\beta$-geo, under the control of the Ptpn23 promoter (Nord et al., 2006). Our results show that Ptpn23 is essential for early mouse embryonic development

\footnotetext{
Abbreviations used in this paper: $\beta$-geo, $\beta$-galactosidase-neomycinphosphotransferase II; BRO, BRO1-like domain; E, embryonic day; HD-PTP, His-domain-containing protein tyrosine phosphatase; HIS, histidine-rich domain; PTP, protein tyrosine phosphatase; PTPN23, Protein-tyrosinephosphatase-n23.
}

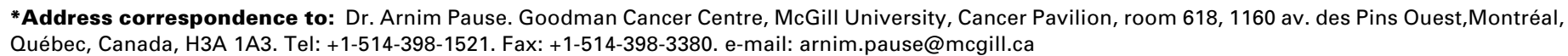

Supplementary Material for this paper is available at: http://dx.doi.org/10.1387/ijdb.082820mg

Accepted: 26 January 2009. Published online: 27 February 2009.

ISSN: Online 1696-3547, Print 0214-6282

(C) 2009 UBC Press

Printed in Spain 
A

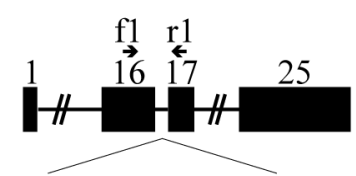

gene-trap insertion

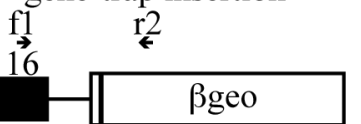

B
C

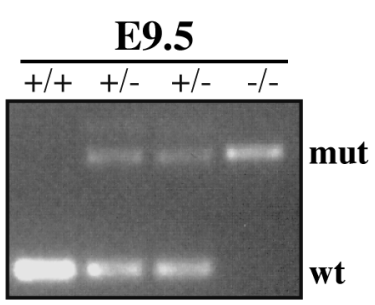

D

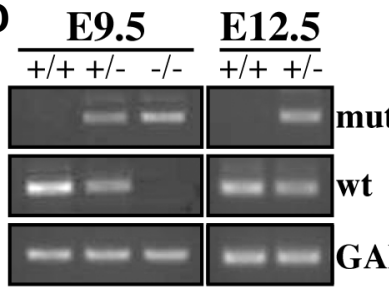

E

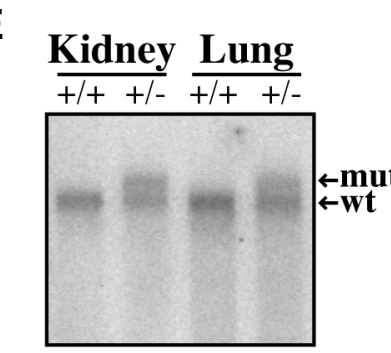

F

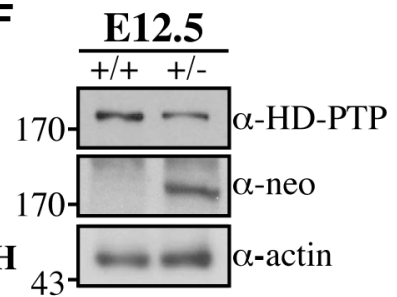

G
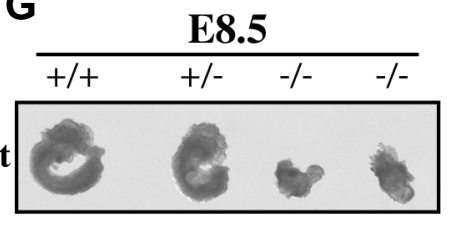

Genotype

H \begin{tabular}{lllll} 
Stage & $\mathbf{N}$ & $+/+$ & $+/-$ & $-/-$ \\
\hline E8.5 & 43 & 15 & 20 & $8^{\mathrm{a}}$
\end{tabular} E9.5 $41 \quad 6 \quad 22 \quad 13^{b}$ E10.5 $48 \quad 17 \quad 26 \quad 5$ b $\begin{array}{lllll}\text { E12.5 } & 24 & 9 & 15 & 0\end{array}$ $\begin{array}{lllll}\text { E16.5 } & 15 & 4 & 11 & 0\end{array}$ postnatal $\begin{array}{lllll}159 & 63 & 96 & 0\end{array}$ a developmentally retarded $b_{\text {necrotic embryos }}$

Fig.1. Generation of a Ptpn23 disrupted mouse. (A) Schematic representation of the 25 exons of the mouse Ptpn23 locus and the $\beta$-galactosidaseneomycin phosphotransferase /I ( $\beta$-geo) gene-trap insertion site between exons 16 and 17. Forward (f1) and reverse ( $r 1$ and $r 2)$ primers used for genotyping are indicated. (B) Schematic representation of the $185 \mathrm{kDa}$ mouse HD-PTP protein and its domains (upper panel) and the predicted 194 kDa gene-trap fusion protein (lower panel). Numbers correspond to amino acids and the location of the probe used for the northern blot is indicated. BRO: BRO1-like domain, HIS: histidine-rich domain, PTP: protein-tyrosine-phosphatase domain, P: proteolytic degradation targeting sequence also know as PEST motif. (C) DNA genotyping at embryonic day (E) 9.5, using primers amplifying the mutated (mut) or the wild type (wt) allele. (D) RTPCR performed on RNA extracts from embryos (E9.5 or E12.5) using primers amplifying the mutated (mut), the wild type (wt) and the endogenous GAPDH transcripts. (E) Northern blot analysis performed on adult kidney and lung RNA extracts using a probe that recognizes both wild type (wt) and mutated (mut) transcripts. (F) Western blot analysis of E12.5 Ptpn23 $3^{+/+}$and Ptpn23+/ embryos using anti-HD-PTP and anti-neomycin ( $\alpha$-neo) antibodies. Actin levels were monitored as loading control. (G) Representative pictures of E8.5 Ptpn23 ${ }^{+/}$, Ptpn23 $3^{+/}$or Ptpn23\% embryos. (H) Detailed genotyping analysis of embryos. $N$, number of genotyped individuals.

since the homozygous deletion of the region encoding the HIS, PTP and PEST domains is lethal around embryonic day 9.5. Furthermore, this model allowed us to characterize the Ptpn23 expression pattern during development and in adult tissues. Here we show that even though Ptpn23 is widely expressed, its expression is not uniform but rather restricted to epithelial cells of several organs such as kidney, lung, digestive tract, liver and skin.

\section{Results and Discussion}

\section{Generation of a Ptpn23 mutant mouse}

We generated a Ptpn23 mutant mouse using an embryonic stem cell clone containing an in-frame $\beta$-galactosidase-neomycin-phosphotransferase II ( $\beta$-geo) insertion between exons 16 and 17 of the mouse Ptpn23 locus (Fig. 1A) (Nord et al., 2006). This gene-trap insertion leads to the expression of a fusion protein encompassing mouse HD-PTP amino acid residues 1 to 548 (out of 1692 amino acids) fused to $\beta$-geo under the control of the Ptpn23 promoter (Fig. 1B). Chimeric mice were generated, germ line transmission was confirmed by PCR and genomic DNA sequence analysis using a $\beta$-geo specific primer confirmed the location of the insertion (not shown). Heterozygous mice were intercrossed and the Mendelian transmission of the $\beta$-geo cassette insertion between exons 16 and 17 was verified by PCR (Fig. 1C) and RT-PCR (Fig. 1D). For simplicity, we will refer to the gene-trapped allele as null (-). We detected wild type $\left(\right.$ Ptpn23 $\left.3^{+/+}\right)$, heterozygous $\left(\right.$Ptpn23 $\left.3^{+/}\right)$and homozygous (Ptpn23 ${ }^{-/}$) embryos at embryonic day (E) 9.5 (Fig. 1 C,D) but only Ptpn23 $3^{+/+}$and Ptpn23 $3^{+-}$at E12.5 (Fig. 1D). Northern blot analysis of the Ptpn23 RNA transcripts expressed in adult kidney and lung extracts confirmed the presence of a full length Ptpn23 wild type (wt) transcript in Ptpn23+/+ organs and the existence of a second larger transcript in the Ptpn23 $3^{+-}$organs, corresponding to the fusion of the Ptpn23 exons 1-16 with the $\beta$-geo cassette (Fig. 1E). Finally, western blot analysis using an antibody generated against the HD-PTP HIS domain, absent in the gene-trap fusion protein (see Fig. S1 for the characterization of the antibody), shows a decrease in the HD-PTP protein level in the Ptpn23+- E12.5 embryos (Fig. 1F). As expected, an anti-neomycin ( $\alpha$-neo) antibody recognizes a band only in the Ptpn23+/- sample at the expected size of the fusion protein. All together, these data confirm that the gene-trap cassette is inserted between exons 16 and 17, is transcribed in fusion with the 5' portion of Ptpn23and that a HD-PTP (amino acids 1-548)$\beta$-geo fusion protein is expressed.

\section{Deletion of the 3' portion of the Ptpn23 gene leads to embryonic lethality}

Since we never found Ptpn23/- embryos at postnatal stages $(n=159)$, detailed genotyping analysis were performed on embryos obtained from Ptpn23 $3^{+/-}$intercrosses (Fig. $1 \mathrm{G}, \mathrm{H}$ ). Although we did not detect any Ptpn23-1- embryos after E12.5, interestingly we found Ptpn23 ${ }^{-/}$embryos as disorganized mass of cells surrounded by maternal hemorrhagic tissue at E9.5 and E10.5 (Fig. 1H). None of the Ptpn23\% embryos $(n=18)$ showed recognizable embryonic structures at E9.5 and E10.5. In addition, all the Ptpn23 ${ }^{-1}$ embryos found at E8.5 $(n=8)$ were 
significantly smaller than normal embryos and their appearance were similar to E6.5 or early E7.5 (late egg cylinder stage) but never reached the E8.5 stage (Fig. $1 \mathrm{G}, \mathrm{H}$ ). These observations indicated the importance of Ptpn23functions during early embryogenesis before E8.5. However, as expected from Mendelian inheritance ratios, a total of $21 \%(26 / 132)$ of embryos were detected as Ptpn23-- between E8.5 to E10.5, suggesting the fertilization and implantation ( E4.5) steps were not affected in Ptpn23-/- embryos. Thus, our results suggest that Ptpn23plays a critical role in early stages of development between E4.5 E7.5, where the period of gastrulation takes place and embryonic ectoderm differentiates to form the neural plate.

\section{Developmental expression profile of Ptpn23}

While Ptpn23 expression was described as ubiquitous in adult human and rat organs by northern blot analysis, its cell type and developmental expression profile has never been described (Cao et al., 1998; Mariotti et al., 2006). Since the $\beta$ galactosidase reporter is expressed under the control of the

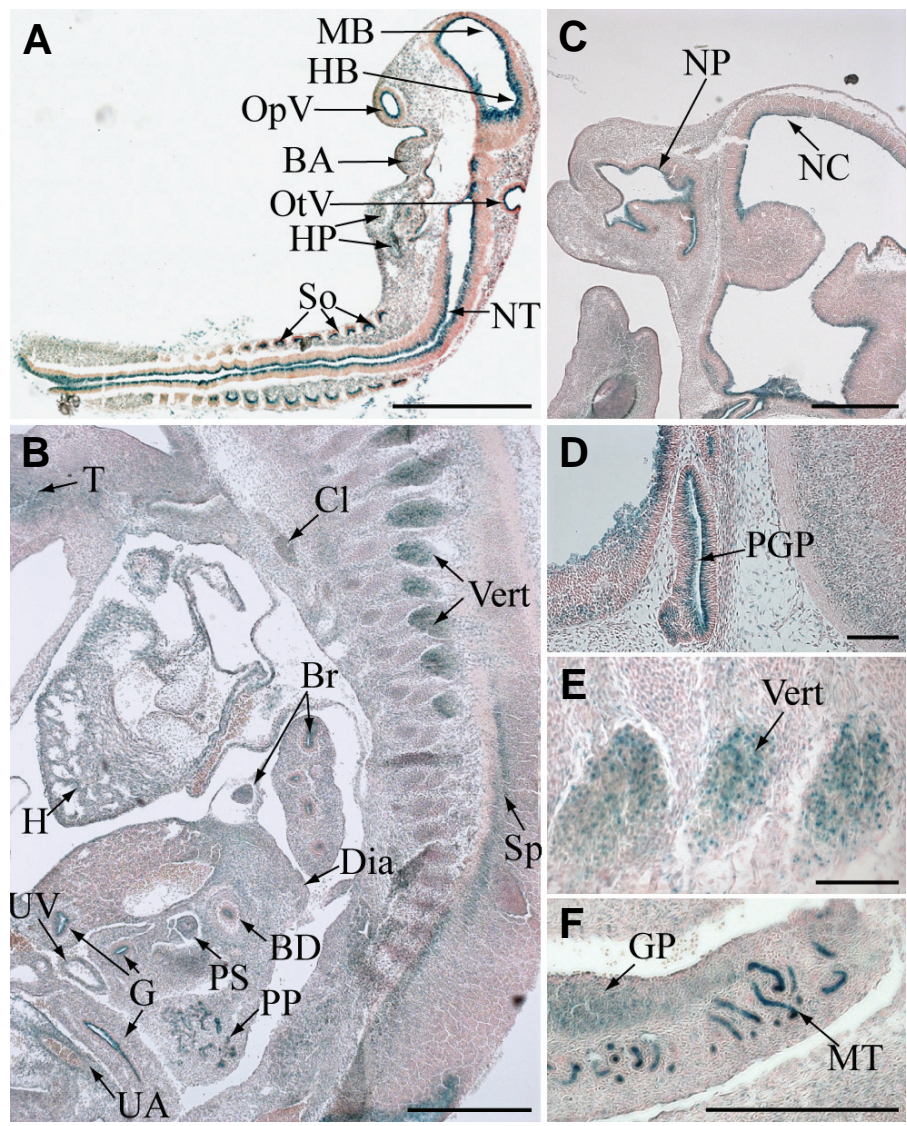

endogenous Ptpn23promoter, we took advantage of our mouse model to characterize the Ptpn23 expression profile during embryonic development and in adult tissues. To proceed, we performed X-Gal staining on heterozygous animal sections, simultaneously with wild-type animals to control for the specificity of the staining.

We first determined the expression of Ptpn23at E9.5, as the Ptpn23 homozygous deletion induces embryonic lethality around that stage. We observed a predominant Ptpn23 expression in the nervous system, especially in the neuroepithelial lining of the midbrain (Fig. 2A; MB), hindbrain (Fig. 2A; HB), at the inner side of the optic (Fig. 2A; OpV) and otic (Fig. 2A; OtV) vesicles
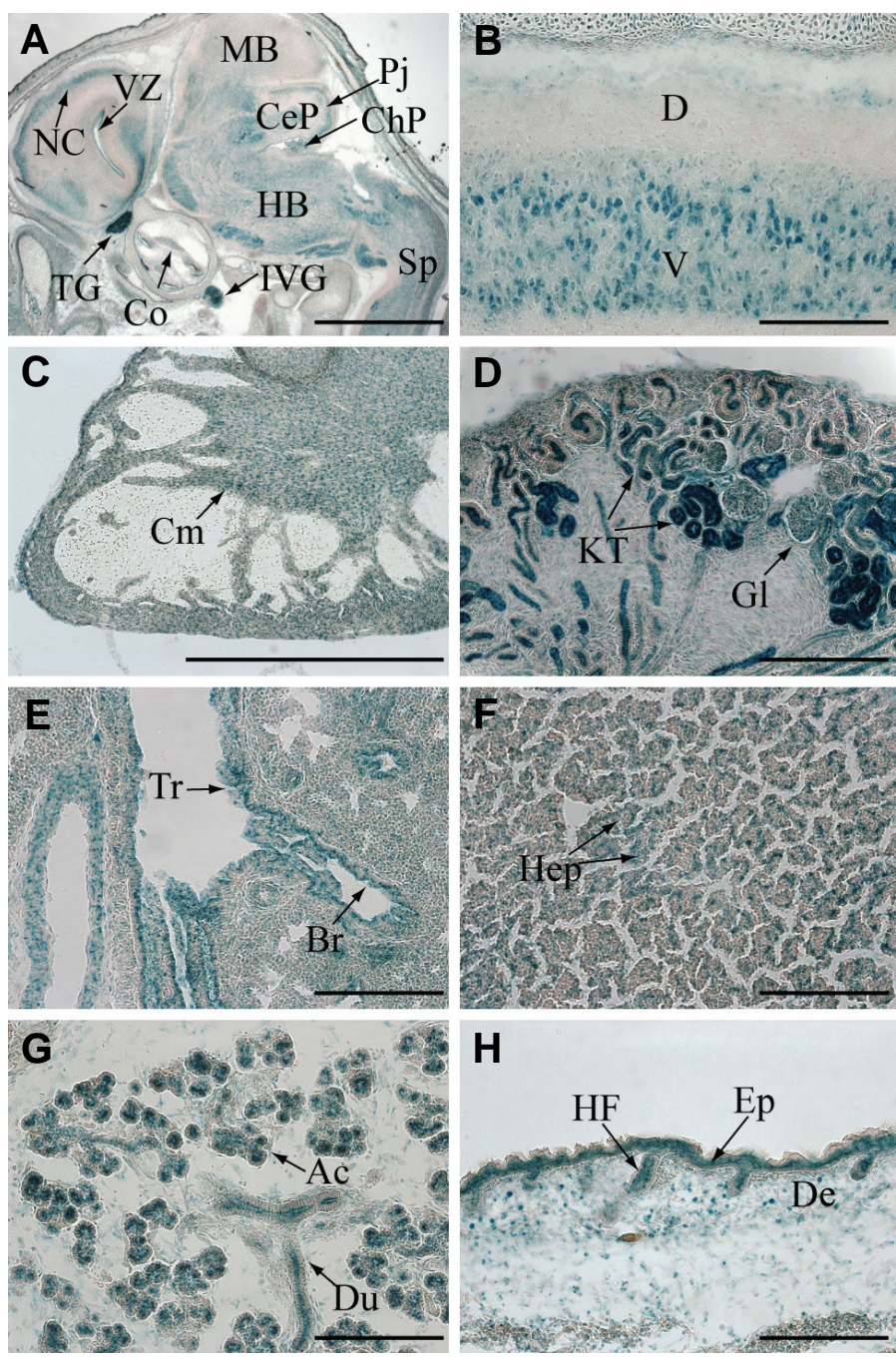

$\mathrm{H}$

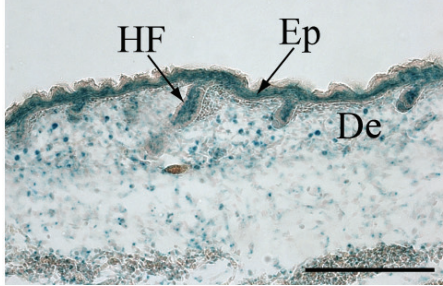

Fig. 2 (Left). Early expression profile of Ptpn23. X-Gal staining of sagittal cryosections of Ptpn23 ${ }^{+/}$embryos corresponding to areas of Ptpn23 expression at E9.5 (A) and E12.5(B-F). See text for details. Bars: A,B, $500 \mu \mathrm{m} ; \mathrm{D}-\mathrm{F}, 100 \mu \mathrm{m}$. BA, branchial arches; BD, bile duct; Br, bronchia; Cl, Clivus; Dia, diaphragm; G, gut; GP, gonad primordium; H, heart; HB, hindbrain; HP, heart precursor; MB, midbrain; MT, mesonephric tubules; NC, neopallial cortex; NP, nasopharynx; NT, neural tube; OpV, optic vesicle; OtV, otic vesicle; PGP, pituitary gland precursor; PP, pancreatic primordium; PS, precursor of the stomach; So, somites; Sp, spinal cord; T, tongue; UA, umbilical artery; UV, umbilical vein; Vert, Vertebrae.

Fig. 3 (Right). Expression pattern of Ptpn23 in late embryonic development. Ptpn23 expression detected by X-Gal staining performed on Ptpn23+l-E16.5 sagittal cryosections. See text for details. Bars: A, $2 \mathrm{~mm} ; B-H, 200 \mu \mathrm{m}$. Ac, acini of the submandibular gland; Br, bronchia; CeP, cerebellum; ChP, choroids plexus; Cm, cardiomyocytes; Co, cochlea; De, dermis; D, dorsal; Du, duct of the submandibular gland; Ep, epidermis; Gl, glomeruli; HB, hindbrain; Hep, hepatocytes; HF, hair follicles; IVG, inferron vagal ganglion; KT, kidney tubules; MB, midbrain; NC, neopallial cortex; Pj, Purkinje cell layer; Sp, spinal cord; TG, trigeminal ganglion; Tr, trachea; V, ventral; VZ, ventricular zone. 
and in the ventricular layer of the forebrain (not shown). Strong expression is also observed in the neuroepithelium of the neural tube (Fig. 2A; NT), in somites (Fig. 2A; So) and faint expression is observed in branchial arches (Fig. 2A; BA) and heart precursor (Fig. 2A; HP). At E12.5, Ptpn23shows a wider expression, precisely in the tongue $(T)$, heart $(H)$, cartilage primordium of the clivus $(\mathrm{Cl})$ and the vertebrae (Vert), epithelium of the lung bronchia $(\mathrm{Br})$, gut $(\mathrm{G})$, precursor of the stomach (PS) and common bile duct (BD) (Fig. 2 B,E). Strong expression is also observed in the spinal cord $(\mathrm{Sp})$, pancreatic primordium $(\mathrm{PP})$, endothelial cell layer of the umbilical vein (UV), artery (UA) and diaphragm (Dia) (Fig. 2B). Pronounced Ptpn23 expression is detected in the neuroepithelium of the neopallial cortex (NC), epithelium of the nasopharynx (NP) (Fig. 2C), pituitary gland precursor (PGP) (Fig. 2D), gonad primordium (GP) and mesonephric tubules (MT) (Fig. 2F).

Later during development (E16.5), Ptpn23 expression is detected in most of the organs, however some cell types and structures described below strongly expressed Ptpn23, suggesting a non-uniform expression (Fig. 3). Indeed, Ptpn23 is markedly expressed in multiple brain regions such as the neopallial cortex (NC) and its ventricular zone (VZ), midbrain $(\mathrm{MB})$, hindbrain $(\mathrm{HB})$, Purkinje cell layer $(\mathrm{Pj})$ of the cerebellum (CeP), choroids plexus (ChP), internal side of the cochlea (Co), as well as trigeminal (TG) and inferior vagual (IVG) ganglions (Fig. 3A). Marked Ptpn23 expression is also observed in cells at the ventral side $(\mathrm{V})$ of the spinal cord, which are probably motoneurons (Fig. 3A; Sp, B) but not at the dorsal side (D). Consistent with earlier stages, high Ptpn23 expression is detected in cardiomyocytes ( $\mathrm{Cm}$ ) of the heart (Fig. 3C) and in tubules (KT) and glomeruli (Gl) of the kidney cortex (Fig. 3D). Moreover, substantial expression is observed in epithelial cells of several organs such as the epithelium of the lung trachea (Tr) and bronchia (Br) (Fig. 3E), liver hepatocytes (Fig. 3F, Hep), acini (Ac) and ducts (Du) of the submandibular salivary gland (Fig. 3G) and inner layer of the hair follicles (HF) as well as the epidermal (Ep) and the dermal (De) layer of the skin (Fig. $3 \mathrm{H}$ ). Thus, the embryonic expression pattern shows that Ptpn23 is expressed during all stages of organogenesis and is highly enriched in epithelia.

\section{Expression pattern of Ptpn23 in adult mouse organs}

To determine if the developmental expression pattern of Ptpn23is preserved after birth, we investigated its expression in adult organs (Fig. 4). Results show that Ptpn23 expression persists at the adult stage and is observed in several brain regions such as cerebral cortex (CC), hypothalamus ( $\mathrm{Hp})$ and thalamus (Th) (Fig. 4A). Ptpn23is also substantially expressed in the Purkinje cells $(\mathrm{Pj})$ layer of the cerebellum and some intensely stained cells in addition with uniform and faint staining are detected in the granular layer (GrL) (Fig. 4B). Strongest expression is detected in the renal tubules of the kidney cortex (KT), but in contrast with the E16.5 pattern, no expression was observed in glomeruli (GI) (Fig. 4C). Similarly to the E16.5 expression profile, Ptpn23expression is detected in the epithelial cells of the trachea (not shown), bronchia (Br) (Fig. 4D), hair follicles (HF), epidermal layer (Ep) of the skin (Fig. 4E) and hepatocytes (Hep) of the adult liver (Fig. 4F). Moreover, pronounced and specific expression has been detected in the epithelium (Epi) of the stomach (Fig. 4G), small intestine (not shown) and colon (Fig. $4 \mathrm{H}$ ). We did not observe $\beta$-gal expression in adult heart, spleen and muscle, suggesting a weak expression below detection limit or an absence of Ptpn23 expression in these tissues (not shown).

In summary, we demonstrate an essential role for Ptpn23 during mouse development, since the homozygous inactivation by gene-trap insertion leads to embryonic lethality around E9.5. Because we observed abnormal but late cylinder stage in Ptpn23\%- embryos at E8.5, deletion of Ptpn23 might affect the process of neurulation, which normally occurs after gastrulation around E7.5. Indeed, our results demonstrate that Ptpn23 is expressed extensively in ectoderm-derived tissues such as neuroepithelium at E9.5, implicating a function in neurulation. Alternatively, absence of Ptpn23 may lead to defects in circulatory system development. Interestingly, Ptpn23 expression has been reported to be upregulated by the angiogenic factor

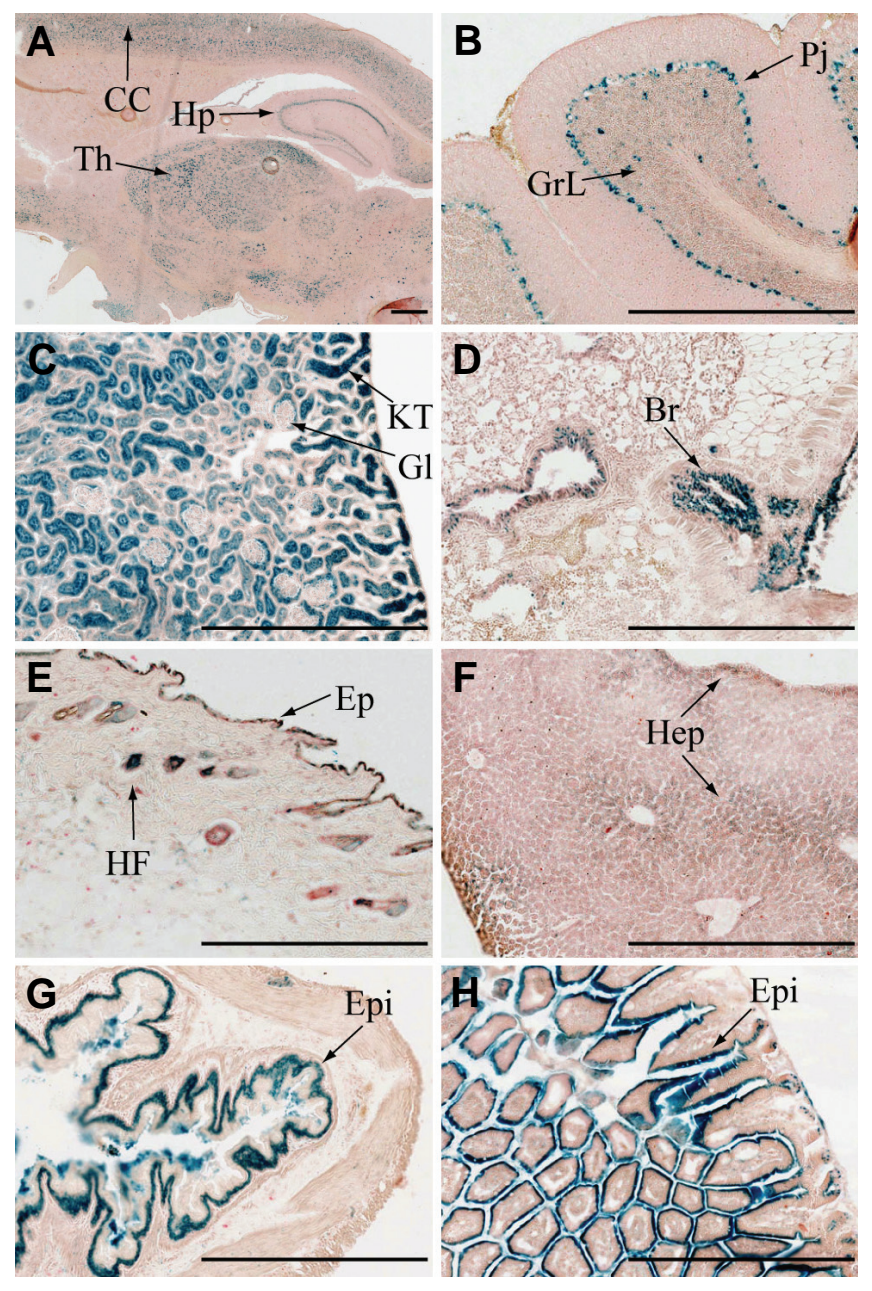

Fig. 4. Ptpn23 expression pattern in adult mouse organs. $X-G a l$ staining performed on cryosections of brain (A), cerebellum (B), kidney (C), lung (D), skin (E), liver (F), stomach (G) and colon (H) from 6 month old Ptpn23 $3^{+-}$mice, corresponding to the location of Ptpn23 expression. See text for details. Bars, $500 \mu \mathrm{m}$. CC, cerebral cortex; Hp, hypothalamus, Th, thalamus; GrL, granular layer of the cerebellum; Pj, Purkinje cell layer of the cerebellum; KT, kidney tubules; GI, glomeruli; Br, bronchia; HF, hair follicle; Ep, epidermis; Hep, hepatocytes; Epi, epithelium. 
Tat and to regulate endothelial cell migration, suggesting a potential role in angiogenesis (Mariotti et al., 2006; Castiglioni et al., 2007; Mariotti et al., 2008). The gene-trap insertion results in a protein containing the complete BRO1-like domain fused to $\beta$-geo. This insertion leads to a deletion of the HIS, PTP and PEST domains of HD-PTP. Interestingly, the tumor suppressor activity of the rat ortholog of HD-PTP in ras-mediated $\mathrm{NIH}$-3T3 transformation was abrogated when a protein truncated for the PTP and PEST domains was expressed (Cao et al., 1998). Moreover, the expression of a truncated HD-PTP protein containing only the BRO1-like domain result in a nonfunctional protein with respect to intracellular sorting of endocytosed cargo and tumor suppression, suggesting that the C-terminal domains of HD-PTP are essential for these activities (Doyotte et al., 2008; Gingras et al. unpublished data). PTPN23 expression has been previously described as ubiquitous in adult human and rat tissues (Cao et al., 1998; Mariotti et al., 2006). Our detailed histological analysis demonstrates that Ptpn23 is widely expressed during mouse embryonic development and in adulthood however its expression pattern is restricted to specific cell types. In fact, Ptpn23 is preferentially expressed in the epithelium of multiple organs and marked Ptpn23 expression has been detected in the renal tubules, in the lung bronchia and in multiple brain compartments. Interestingly, most of the renal, lung and cervical tumors that are frequently deficient for the 3p21.3 tumor suppressor cluster where HD-PTP is encoded, originates from epithelial cells that strongly expressed HD-PTP. The specificity of HD-PTP expression in epithelia suggests that it could be involved in signaling pathways activated in epithelial cells. Consistently, a role for HD-PTP in the endosomal transport of the EGF receptor, which is highly expressed in tissues from epithelial and neuronal origins, and resulting in the enhancement of its downstream signaling has recently been described (Miura et al., 2008).

\section{Materials \& Methods}

\section{Ptpn23 mouse model}

The mouse YHB-130 embryonic stem cell line containing an inframe $\beta$-geo ( $\beta$-galactosidase and neomycin phosphotransferase II fusion) insertion between exons 16 and 17 of the mouse Ptpn23 gene was obtained from the International Gene-Trap Consortium (www.genetrap.org). The YHB-130 clone (129 s/v background) was injected into $\mathrm{C} 57 \mathrm{BL} / 6$ blastocysts, chimeric mice were generated and intercrossed to obtain Ptpn23 $3^{+-}$mice. For the embryonic genotyping analysis, Ptpn23 ${ }^{+/-}$mice were mated and embryos at different stages were dissected in cold PBS and the yolk sacs were kept for genotyping analysis (described below). Representative pictures of E8.5 Ptpm23 +1 ${ }^{+}$, Ptpn23 $3^{+-}$and Ptpm23 ${ }^{-/}$embryos were taken using a Stemi SV11 stereomicroscope equipped with a CCD camera (Zeiss).

\section{Genotyping, RT-PCR, Northern blot and Western blot analyses}

Genomic DNA or RNA was isolated from tail biopsies, complete embryos or yolk sacs and PCR or RT-PCR were performed using a forward primer located in Ptpn23 exon 16 and reverse primers located in Ptpn23 exon 17 or $\beta$-geo. For RT-PCR analysis, GAPDH was amplified as control of the RNA levels. Primer sequences are available in figure S2. Northern blot analysis was performed on Ptpn23 $3^{+/+}$or Ptpn23 $3^{+-}$adult kidney and lung RNA extracts using a human cDNA PTPN23 probe (human amino acids 231 to $403=98 \%$ homology with mouse sequence) that binds specifically to both wild type and mutant transcripts. Western blot analyses were performed on protein extracts from E12.5 Ptpr23 $3^{++}$and Ptpr23 $3^{+-}$embryos using our specific antiHD-PTP rabbit polyclonal antibody generated against its HIS domain (human amino acids 400 to 800 ) that only recognizes the wild type protein, an anti-neomycin antibody ( $\alpha$-neo: rabbit polyclonal antineomycin phosphotransferase II antibody, Upstate) and an anti- $\beta$-actin antibody (mouse monoclonal AC74, Sigma-Aldrich) as loading control.

\section{$X$-Gal staining}

Ptpn23+/- females were mated with C56BL/6 males, embryos were dissected and yolk sacs were used for genotyping as described above. Dissected Ptpn23+/+ or Ptpn23+/- adult tissues (6 months) or complete embryos were fixed $(0,2 \%$ glutaraldehyde) and embedded in OCT (Tissue-Tek). The E16.5 embryos were manually hemisected halfway and arm and legs were removed to permit efficient fixation. Frozen tissues and embryos were sectioned into $10 \mu \mathrm{m}$ slices; the $\beta$-galactosidase activity was detected by X-Gal staining and counter stained with Nuclear Fast Red (Sigma-Aldrich) according to the instructions of the International Gene-trap consortium. Staining was terminated before background signal was detected in Ptpn23 +/+ samples and was performed on three to five different individuals from each genotype at each stage. Representative pictures were taken using a XT Scan Scope Imaging System (Aperio).

\section{Acknowledgements}

We thank J. Penney and E. Migon from the McGill Transgenic Mouse Core Facility for the mouse model generation. We are grateful to M. Narlis from the McGill Cancer Centre Developmental Histology Facility for sample sectioning, to D. Grote for helpful discussions and protocols and to $S$. Welbourn and AB. Dydensborg for critical reading of this manuscript. MCG was supported by a CIHR McGill University Cancer Consortium fellowship and a fellowship from Fond de la recherche en santé du Québec (FRSQ) and holds a Kidney Foundation of Canada fellowship. DK holds a CIHR McGill University Cancer Consortium studentship and a Maysie MacSporran graduate studentship award. MB holds a Canada Research Chair in Developmental Genetics of the Urogenital System, MLT a Jeanne and Jean-Louis Lévesque Chair in Cancer Research and a NCIC/CCS operating grant and AP a Canada Research Chair in Molecular Oncology.

\section{References}

CAO, L., ZHANG, L., RUIZ-LOZANO, P., YANG, Q., CHIEN, K.R., GRAHAM, R.M. and ZHOU, M. (1998). A novel putative protein-tyrosine phosphatase contains a BRO1-like domain and suppresses Ha-ras-mediated transformation. J Biol Chem 273: 21077-21083.

CASTIGLIONI, S., MAIER, J.A. and MARIOTTI, M. (2007). The tyrosine phosphatase HD-PTP: A novel player in endothelial migration. Biochem Biophys Res Commun 364: 534-539.

DOYOTTE, A., MIRONOV, A., MCKENZIE, E. and WOODMAN, P. (2008). The Bro1-related protein HD-PTP/PTPN23 is required for endosomal cargo sorting and multivesicular body morphogenesis. Proc Natl Acad Sci USA 105: 6308-6313.

HESSON, L.B., COOPER, W.N. and LATIF, F. (2007). Evaluation of the 3p21.3 tumour-suppressor gene cluster. Oncogene 26: 7283-7301.

IMREH, S., KLEIN, G. and ZABAROVSKY, E.R. (2003). Search for unknown tumor-antagonizing genes. Genes Chromosomes Cancer 38: 307-321.

JI, L., MINNA, J.D. and ROTH, J.A. (2005). 3p21.3 tumor suppressor cluster: prospects for translational applications. Future Onco/1: 79-92.

MARIOTTI, M., CASTIGLIONI, S., GARCIA-MANTEIGA, J.M., BEGUINOT, L. and MAIER, J.A. (2008). HD-PTP inhibits endothelial migration through its interaction with Src. Int J Biochem Cel/ Bio/41: 687-693.

MARIOTTI, M., CASTIGLIONI, S. and MAIER, J.A. (2006). Expression analysis and modulation by HIV-Tat of the tyrosine phosphatase HD-PTP. $J \mathrm{Cell}$ Biochem 98: 301-308. 
MIURA, G.I., ROIGNANT, J.Y., WASSEF, M. and TREISMAN, J.E. (2008). Myopic acts in the endocytic pathway to enhance signaling by the Drosophila EGF receptor. Development 135: 1913-1922.

NORD, A.S., CHANG, P.J., CONKLIN, B.R., COX, A.V., HARPER, C.A., HICKS, G.G., HUANG, C.C., JOHNS, S.J., KAWAMOTO, M., LIU, S. et al. (2006). The International Gene Trap Consortium Website: a portal to all publicly available gene trap cell lines in mouse. Nucleic Acids Res 34: D642-D648.

TOYOOKA, S., OUCHIDA, M., JITSUMORI, Y., TSUKUDA, K., SAKAI, A., NAKAMURA, A., SHIMIZU, N. and SHIMIZU, K. (2000). HD-PTP: A novel protein tyrosine phosphatase gene on human chromosome 3p21.3. Biochem Biophys Res Commun 278: 671-678.

\section{Further Related Reading, published previously in the Int. J. Dev. Biol.}

See our recent Special Issue Fertilization, in honor of David L. Garbers and edited by Paul M. Wassarman and Victor D. Vacquier at: http://www.ijdb.ehu.es/web/contents.php?vol=52\&issue=5-6

Activated Ras induces lens epithelial cell hyperplasia but not premature differentiation

Lixing W. Reneker, Leike Xie, Li Xu, Venkatesh Govindarajan and Paul A. Overbeek Int. J. Dev. Biol. (2004) 48: 879-888.

Receptor protein tyrosine phosphatase mu expression as a marker for endothelial cell heterogeneity; analysis of RPTPmu gene expression using LacZ knock-in mice. Elianne A Koop, Susana M Chuva de Sousa Lopes, Elles Feiken, Hans A R Bluyssen, Martin van der Valk, Emile E Voest, Christine L Mummery, Wouter H Moolenaar and Martijn F B G Gebbink. Int. J. Dev. Biol. (2003) 47: 345-354.

Receptor protein-tyrosine phosphatase signalling in development.

J den Hertog, C Blanchetot, A Buist, J Overvoorde, A van der Sar and L G Tertoolen Int. J. Dev. Biol. (1999) 43: 723-733.

A genetic screen for tumor suppressor genes in the mouse.

A Bradley. Int. J. Dev. Biol. (1996) 40: S59-S60.

Tumor suppressor genes as negative growth regulators in development and differentiation.

D H Gutmann. Int. J. Dev. Biol. (1995) 39: 895-908.
5 yr ISI Impact Factor $(2008)=3.271$

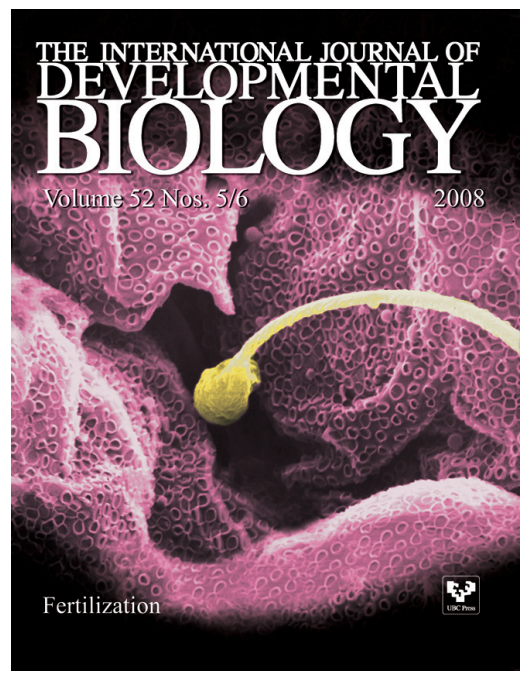

